\title{
Screening and Analysis of Thyroid-Disrupting Chemicals Based on Selective Recognition for a Thyroxine-Binding Peptide
}

\author{
Xiaoya Zhao ${ }^{1}$, Han Wang ${ }^{1}$, Yinzhu Shang ${ }^{1}$, Peng Wang ${ }^{1}$, Danqi Cheng ${ }^{2}$, \\ Zhijia Zhuang ${ }^{2}$, Tao Jing, ${ }^{2, *}$ \\ ${ }^{1}$ 15\#Jinyinhu Road, Dongxihu District, Technology, Center of the Wuhan Customs, Wuhan, Hubei, \\ 430050, P.R. China; \\ ${ }^{2}$ State Key Laboratory of Environment Health (Incubation), Key Laboratory of Environment and \\ Health, Ministry of Education, Key Laboratory of Environment and Health (Wuhan), Ministry of \\ Environmental Protection, School of Public Health, Tongji Medical College, Huazhong University of \\ Science and Technology, Wuhan, Hubei 430030, P. R. China \\ *E-mail: jingtao@ hust.edu.cn
}

Received: 1 June 2021 / Accepted: 27 July 2021 / Published: 10 September 2021

Thyroxine (TH) is an essential hormone for animal growth and development. Environmental exposure to thyroid-disrupting chemicals (TDCs) can alter the synthesis, secretion and transport of TH. In this study, a peptide with strong affinity for L-thyroxine (T4) was screened and synthesized, followed by attached to a gold electrode surface and then evaluated for its selective recognition ability for 36 chemicals with known activities towards TTR. The peptide-Au electrode could distinguish TCDs and thyroid-nondisrupting chemicals (non-TCDs) by impedance. The sensitivity, specificit and correct assignment of TDC action for these test compounds were 61.5, 73.9 and 69.4\%, respectively. This method can therefore be used as a rapid, specific and cost effective way to identify TCDs.

Keywords: Thyroxine (TH); Thyroid-Disrupting Chemicals(TDCs); Peptide; Gold electrode; Impedance

\section{FULL TEXT}

(C) 2021 The Authors. Published by ESG (www.electrochemsci.org). This article is an open access article distributed under the terms and conditions of the Creative Commons Attribution license (http://creativecommons.org/licenses/by/4.0/). 